\title{
«Ich möchte ihm lieber zehen Pferde als meine Frau zur Kur überlassen»
}

\author{
Friedrich Schiller war wohl der einzige Medizinstudent, der drei Examensarbeiten \\ schreiben musste. Eigentlich wollte er ja Theologe werden, doch der Herzog be- \\ stimmte es anders. Dennoch studierte Schiller Medizin «con amore». Nachtwachen \\ nutzte er aber, um die «Räuber» zu schreiben.
}

«Der Starke ist am mächtigsten allein.»

«Nacht muss es sein, wo Friedlands Sterne strahlen.» «Ernst ist das Leben, heiter ist die Kunst.»

Sätze wie in Granit gehauen. Schiller natürlich, der wortgewaltigste unter den Klassikern. Eigentlich hatte er Theologe werden wollen; dann sollte er Jurist werden und schliesslich wurde er Arzt und Dichter.

Johann Christoph Friedrich Schiller wurde am 8. November 1759 in Marbach/Neckar geboren. Sein Vater war gelernter Barbier und hatte es bei der Armee zum Wundarzt und Hauptmann gebracht. Das Studium der Theologie, das ihm nicht vergönnt gewesen war, sollte der Sohn absolvieren [1]. Doch Herzog Carl Eugen von Württemberg wollte es anders: «In meiner Akademie können keine Theologen gebildet werden, Sein Sohn kann sich die Jurisprudenz wählen!» [2].

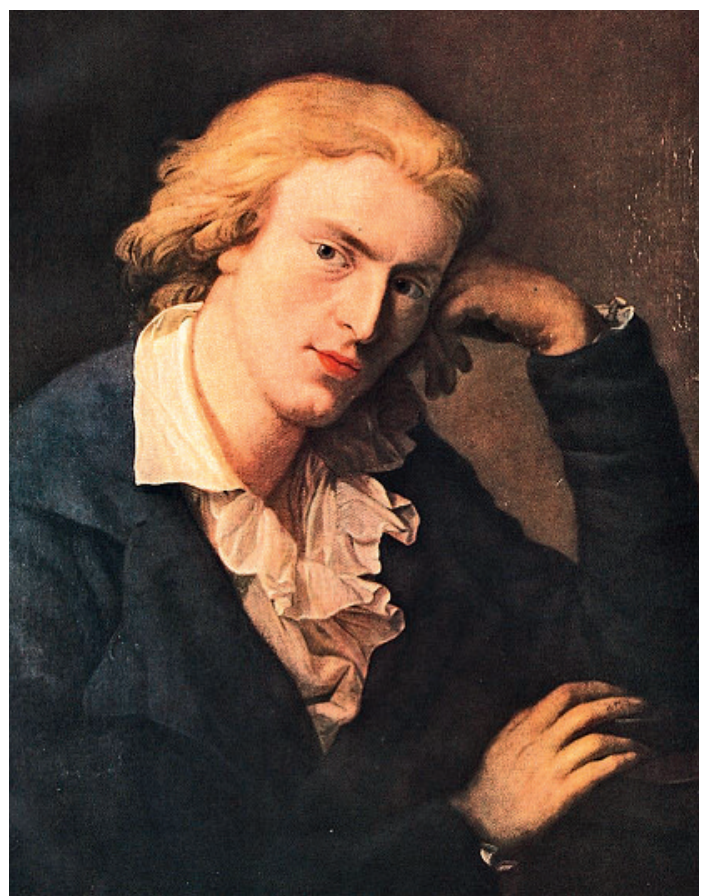

Friedrich Schiller um 1790. Gemälde von Anton Graff.
Die «Akademie», das war die sogenannte Karlsschule bei Stuttgart, in die Schiller im Januar 1773 als 447. Schüler eintrat. Zuvor hatte er seit 1767 die Lateinschule in Ludwigsburg besucht, deren Abschluss die Voraussetzung für die Aufnahme ins Predigerseminar war. Der Wechsel zur Karlsschule geschah nicht freiwillig: Zwei Mal hatte Vater Schiller den Wunsch des Herzogs bereits abgelehnt, seinen Sohn auf die Akademie zu schicken, ein drittes Mal traute er sich nicht. Damit war die theologische Laufbahn beendet.

\section{Die Akademie}

Herzog Carl Eugen von Württemberg hatte im Jahre 1770 eine der modernsten Schulen der Zeit gegründet, die Karlsschule. Eintrittsvoraussetzung war nicht der gesellschaftliche Status, sondern die Leistung. Die Schule sollte eine Kaderschmiede sein, die das Verwaltungspersonal des Herzogtums ausbildete. Der Herzog liess sich von den Lateinschulen regelmässig die Namen der Jahrgangsbesten nennen und wurde so auf Schiller aufmerksam. Seit 1773 hatte die Karlsschule den Status einer Militärakademie, weshalb dort keine Theologen ausgebildet werden konnten.

Schillers Stundenplan im ersten Jahr bestand aus Latein, Griechisch, Französisch, Geschichte, Geographie, Mathematik, Philosophie, Rhetorik und Poetik. Hinzu kam Unterricht im Reiten, Fechten und Tanzen. Das Unterrichtspensum betrug 47 Wochenstunden. Obwohl das Reglement militärisch war, werden die Lehrer als liberal beschrieben. Der Herzog hatte absichtlich junge Universitätsabsolventen ausgewählt, von denen er sich neue, unkonventionelle Unterrichtsmethoden erhoffte. Ab 1774 nahm Schiller am juristischen Fachunterricht teil. Dieser langweilte ihn, so dass er es vorzog, während der Vorlesungen an poetischen Versuchen zu arbeiten. Der literarische Zirkel, dem er und einige Kommilitonen angehörten, hatte ein «Grossprojekt» in Angriff genommen, zu dem Schiller den tragischen Teil beisteuern sollte. Leider ist davon nichts erhalten. Das früheste literarische Zeugnis ist ein für den Herzog verfasster Bericht aus dem Herbst 1774 über die Mitschüler und sich selbst, an dessen 
Ende er erneut seinem Wunsch Ausdruck verleiht, «Gottesgelehrter» zu werden [3].

\section{Medizinstudium}

Daraus wurde jedoch nichts, denn Ende 1775 fiel dem Herzog auf, dass die Akademie zu viele Juristen ausbildete. Einige Schüler mussten zur neugegründeten medizinischen Fakultät wechseln, unter ihnen Schiller. Ob er sich freiwillig dazu gemeldet hat oder einfach zufällig ausgewählt wurde, lässt sich nicht mehr feststellen. Schillers Freund Hoven begründete den Wechsel später mit der Auffassung, dass ihnen die «Medizin mit der Dichtkunst viel näher verwandt zu sein schien als die trockene Jurisprudenz». Andererseits soll Schiller seinem Vater gegenüber geäussert haben, «er könne durchaus diese Laufbahn nicht betreten, er werde sich eher den Tod antun» [4]. Eine Abneigung scheint er gegen die Medizin jedenfalls nicht empfunden $\mathrm{zu}$ haben. Rückblickend schreibt er 1785 sogar, er habe «die Medicin con amore studiert» [5]. Die Verbesserung seiner Zensuren - er war vor dem Fachwechsel der schlechteste Schüler seiner Klasse - deutet darauf hin, dass das stimmt.

Das Medizinstudium begann im Januar 1776. Schiller gehörte zum ersten Jahrgang, der aus nur neun Studenten bestand. Auf dem Lehrplan standen nun wöchentlich je drei Stunden Chemie und Botanik, neun Stunden Pathologie und Therapeutik und je sechs Stunden Anatomie und medizinisches Zeichnen. Hinzu kamen Medizingeschichte, Physiologie, Arzneikunde und Chirurgie, ausserdem Englisch, Religion und Tanzen. Trotz dieses Pensums war noch Zeit für poetische Versuche. Die ersten literarischen Veröffentlichungen stammen aus der Zeit des Medizinstudiums: Von 1776 bis 1780 Gedichte und Vergil-Übersetzungen im «Schwäbischen Magazin», 1782 zusammen mit Kommilitonen die Herausgabe der Sammlung «Anthologie auf das Jahr 1782» [6].

\section{Bei der Abschlussfeier war auch des Herzogs}

\section{neuer Geheimrat anwesend. Sein Name:}

\section{Johann Wolfgang Goethe}

Aus Schillers Studienzeit sind mit Ausnahme der Examensarbeiten zwei medizinische Texte erhalten: Der eine ist das Protokoll der Obduktion eines 17-jährigen Schülers, der an Tuberkulose verstorben war. Der andere besteht aus sieben Berichten über einen selbstmordgefährdeten Kommilitonen, Grammont, dessen psychischen Zustand Schiller vom 26.6.1780 bis 31.7.1780 dokumentierte. In Anlehnung an die von einigen seiner Professoren gelehrte Theorie vermutete er in diesen Berichten einen psychosomatischen Ursprung des Leidens [7]. Es war sein letztes Studienjahr, eigentlich hätte er schon 1779 abschlies-

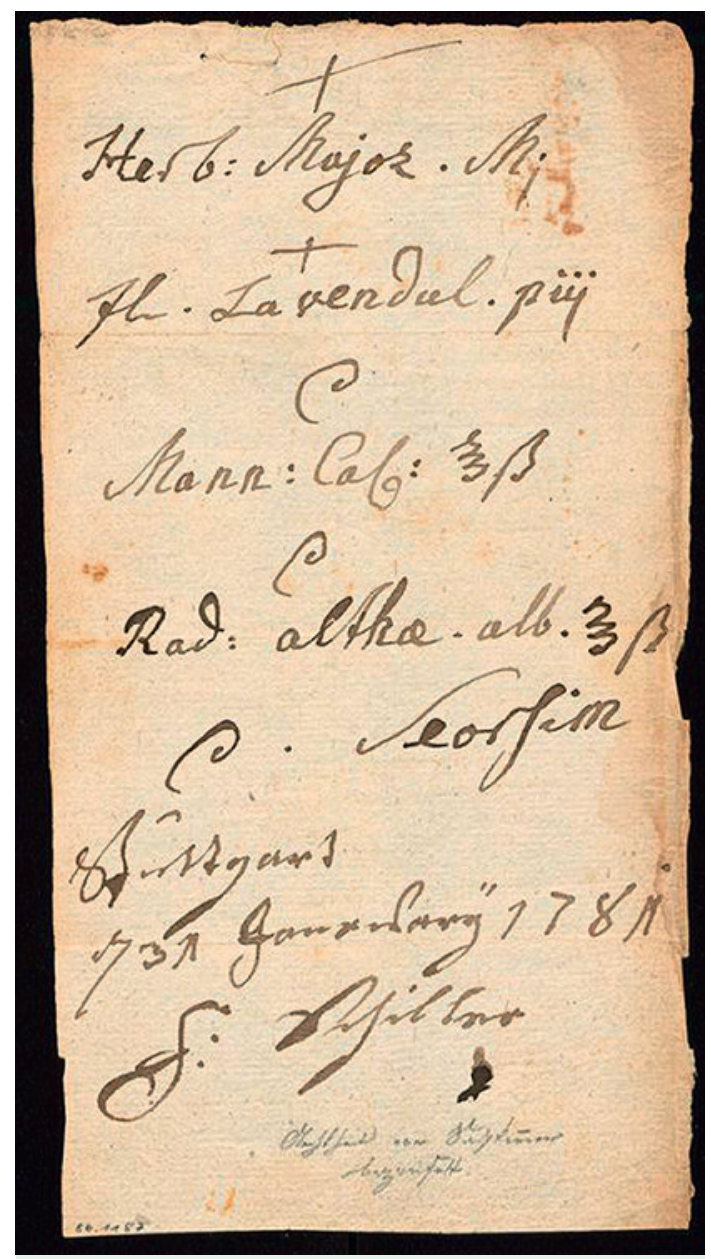

Von Schiller ausgestelltes Rezept.

(c) DLA Marbach, www.dla-marbach.de

sen können. Da die Examensarbeit jedoch abgelehnt worden war, musste er ein Jahr «nachsitzen». Literaturgeschichtlich war das aber kein Nachteil, denn die freie Zeit, wie auch Nachtwachen im Krankenhaus, nutzte Schiller zur Niederschrift der «Räuber». Abgesehen von der abgelehnten Examensarbeit war das Studienjahr 1779 sogar sehr erfolgreich gewesen: drei silberne Medaillen in praktischer Medizin, Arzneikunde und Chirurgie, insgesamt zweitbester des Jahrgangs. Bei der Abschlussfeier am 14.12.1779 war auch des Herzogs neuer Geheimrat anwesend. Sein Name: Johann Wolfgang Goethe. Bis zur ersten persönlichen Begegnung sollte es aber noch 15 Jahre dauern.

\section{Die Examensarbeiten}

Schiller war vermutlich der einzige Medizinstudent, der drei Examensarbeiten schrieb. Die erste reichte er im Herbst 1779 ein. Es handelte sich um eine Abhandlung über die «Philosophie der Physiologie», inspiriert wohl durch den Philosophieunterricht an der Karlsschule. Von dieser Arbeit ist nur eine Zusammenfassung erhalten. Schiller setzte sich mit dem Leib-SeeleProblem auseinander, das er im Unterricht aus den Schriften Descartes (Meditationes de prima philoso- 


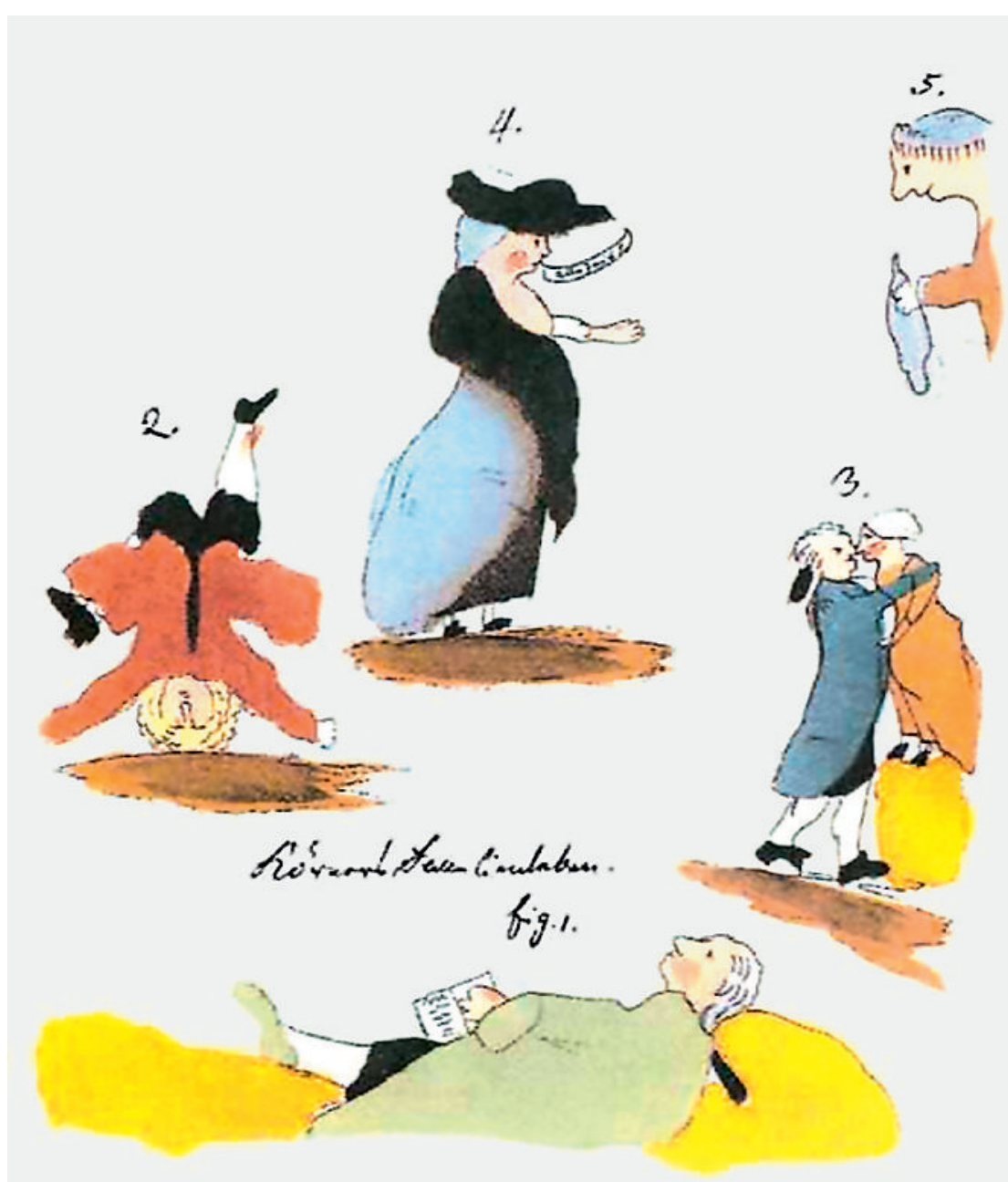

Schiller konnte auch zeichnen: Hier ein Geburtstagsgeschenk für seinen Freund Gottfried Körner. Neben anderen Szenen aus dessen Leben zeigt die Abbildung unten den - bei der Lektüre von Kant - eingeschlafenen Körner. Bei dem auf dem Kopf stehenden Herrn handelt es sich um ein Selbstporträt Schillers.

(๔) Goethezeitportal; www.goethezeitportal.de/index.php?id=3589).

phia), Leibnizens (Discours de métaphysique), Hallers (Primae lineae physiologiae) und La Mettries (L'homme machine) kennengelernt hatte. Die Gutachter lehnten die Arbeit einstimmig ab: zu blumiger Stil, zu konfuse Argumentation und zu gewagte Kritik an den Autoritäten [8]. den Zusammenhang der thierischen Natur des Menschen mit seiner geistigen». Die Fieberschrift wurde abgelehnt. Auf 31 Seiten hatte Schiller sich in 38 Paragraphen mit den Ursachen, den Symptomen und der Therapie der verschiedenen Fieberarten auseinandergesetzt, hatte auch Autoritäten wie Hippokrates und Sydenham sowie die Schriften seiner Lehrer zitiert; die Gutachter bemängelten diesmal, dass dem Autor wesentliche epidemiologische und pathogenetische Details entgangen waren. Er selbst hatte in § 19 zugegeben, nichts über den Einfluss der Luft, der Nahrung und der Ansteckung als Krankheitsursache zu wissen [9].

Die deutschsprachige Abhandlung [10] war quasi eine Neuauflage der ersten Arbeit, also wieder das LeibSeele-Problem, diesmal aber mit Betonung von psychophysischen Wechselwirkungen und mit zahlreichen Zitaten aus der Dramatik, hauptsächlich aus den Werken Shakespeares. Schiller besass sogar die Kühnheit, eine Szene aus den «Räubern» zu zitieren; allerdings war er vorsichtig genug, sie als ein Zitat aus dem Stück «Life of Moor» eines gewissen Krake zu deklarieren. Überraschenderweise akzeptierten die Gutachter diese 39-seitige Arbeit einstimmig. Lediglich der manchmal zu poetische Stil und einige gewagte Spekulationen wurden kritisiert. Damit stand der Entlassung aus der Akademie nichts mehr im Wege.

\section{Regimentsarzt}

Friedrich Schiller hatte bei der Vergabe der Stellen mehr Glück als die meisten seiner Kommilitonen: Einige erhielten gar keine Stelle und wanderten nach Russland aus. Schiller hingegen wurde dem «Regiment Augé» zugeteilt: 420 alte und teilweise invalide Soldaten unter dem Kommando eines 82-jährigen Generals. Wirklich zeitaufwendig war die Tätigkeit nicht: Schiller nahm morgens die Krankmeldungen in der Kaserne entgegen und visitierte dann im Militärhospital, was insgesamt zwei Stunden gedauert haben dürfte. Das liess ihm genügend Zeit, seinen Sold durch eine redaktionelle Tätigkeit für die «Mäntlerische Zeitung» aufzubessern.

Zur Hilfe bei der Ausstellung von Rezepten hatte er 1781 den «Apothekeralmanach» gekauft, das einzige medizinische Werk, das er sich während der Dienstzeit anschaffte. Eines seiner Rezepte ist erhalten:

\section{Die Gutachter lehnten die Arbeit einstimmig ab: zu blumiger Stil, zu konfuse Argumentation und zu gewagte Kritik an den Autoritäten}

Das sollte nicht noch einmal passieren. Deshalb reichte er im November 1780 sicherheitshalber gleich zwei Arbeiten ein: die lateinische Abhandlung «De discrimine febrium inflammatoriarum et putridarum» und den auf Deutsch geschriebenen «Versuch über
Man solle Brechweinstein in Wasser auflösen. Davon sei sogleich die Hälfte einzunehmen.

Im Juli 1781 liess er auf eigene Kosten 800 Exemplare der «Räuber» drucken, fand aber kaum Käufer. In seinem Zimmer «Am langen Graben» in Stuttgart, 
das als «nach Tabak und sonstwie stinkendes Loch» beschrieben wurde, nahmen die Bücher neben einem Haufen leerer Weinflaschen den grössten Platz ein [11].

Zufällig gelangte aber ein Exemplar an Baron Dahlberg, den Intendanten des Mannheimer Nationaltheaters. Er erklärte sich bereit, das Stück zu bringen. Die Uraufführung fand am 13. Januar 1782 statt und war ein Erfolg. Schiller hat sein eigenes Stück anonym im «Wirtembergischen Repertorium», zu dessen Herausgebern er mittlerweile gehörte, rezensiert. Über den Autor - also sich selbst - schrieb er: «Er soll ein Arzt bei einem Wirtembergischen Grenadier-Bataillon sein ... muss er starke Dosen in Emeticis eben so lieben als in Aestheticis, und ich möchte ihm lieber zehen Pferde als meine Frau zur Kur überlassen» [12].

\section{Es gab Beschwerden aus der Schweiz, weil in den «Räubern» Graubünden als «Athen der heutigen} Gauner» bezeichnet wurde

Trotz der erfolgreichen Inszenierung gab es Probleme mit dem Herzog: zweimalige unerlaubte $\mathrm{Ab}-$ wesenheit aus Stuttgart, um bei Vorstellungen dabei zu sein, ausserdem eine Beschwerde aus der Schweiz, weil im 2. Akt der «Räuber» Graubünden als das «Athen der heutigen Gauner» bezeichnet wurde. Zwar machte Schiller geltend, dass er der einzige Absolvent der Akademie sei, der die «Aufmerksamkeit der grossen Welt angezogen» habe [13], dennoch untersagte ihm Carl Eugen weitere nichtmedizinische Veröffentlichungen. Im April 1782 schrieb Schiller an Dahlberg, er habe wenig Hoffnung, seine Neigung zum Drama innerhalb des halben Jahres befriedigen zu können, weil er genötigt sei, eine «medicinische Dissertation» zu schreiben und den «Gradum eines Doctors der Medicin» [14] anzunehmen. Allerdings ist von dieser Dissertation nichts weiter bekannt. Dass er den Ende 1780 in einem Brief mitgeteilten Plan, «Professor in der Physiologie» [15] werden zu wollen, zu diesem Zeitpunkt noch verfolgte, ist angesichts der geringen Erfolgsaussicht aufgrund der in Württemberg herrschenden Ärzteschwemme nicht anzunehmen [16].

Die Aussicht, nie wieder veröffentlichen zu können, bewog ihn schliesslich am 22. September 1782 zur Flucht aus Stuttgart. Damit endet Schillers medizinische Laufbahn - obwohl er wohl zumindest anfangs nicht vorhatte, den Arztberuf aufzugeben. An den ehemaligen Kommilitonen Jacobi schrieb er am 6. November 1782: «Das versteht sich ohnehin, dass ich nur als Medicus Dienst nehme ... weil ich gern hierin etwas vorstellen möchte.» Und am gleichen Tag an die Schwester Christophine: «Ich habe keinen anderen Gedanken, als mein Glück nur allein durch die Medicin zu machen, und werde suchen, innerhalb eines halben Jahrs Doctor zu sein.» Noch drei Jahre später hiess es in einem Brief an den Buchhändler Schwan: «Hier ... bin ich Willens ... mich wieder zu meiner Medicin zu bekehren» [17]. In Wahrheit hat er nie wieder praktiziert und auch nie den Doktorgrad erlangt. Wirklich medizinischen Inhalt in Schillers Werk findet man in den nach der Studienzeit entstandenen Werken nur noch selten. In dem Vortrag «Was kann eine gute stehende Schaubühne eigentlich wirken?» von 1784, in dem Schiller dieser die Aufgabe zuweist, durch «heilsame Leidenschaften» das Blut in «frischere Wallungen» [18] zu treiben, meint man Anklänge an die Hippokratische Humoralpathologie herauszuhören. Sie könnten aber auch indirekt über die Poetik des Aristoteles vermittelt sein, der die Aufgabe der Tragödie darin sieht, durch «Jammer und Schaudern» eine «Reinigung von Erregungszuständen» [19] $\mathrm{zu}$ bewirken.

Sicher hippokratisch ist das Motto der «Räuber»: «Quae medicamenta non sanant, ferrum sanat, quae ferrum non sanat, ignis sanat»- der 87. Aphorismus des Corpus Hippocraticum.

\section{Literatur}

1 Schillers Werke. Nationalausgabe. Weimar: Böhlau. 1943ff., Bd. 42: 3. (im folgenden zitiert als NA und Bandzahl).

2 NA 42: 8.

3 NA 22: 15.

4 NA 42: 8, 10.

5 NA 24: 3.

6 Alt, PA. Schiller. Leben - Zeit - Werk. München: Beck: 2000; Bd. 1: 220-5.

7 NA 22: 17-18, 19-30. Zum Vergleich mit den Berichten anderer Kommilitonen siehe: Kümmel WF. «Mit der Schlange von Epidauros spielen»: Schiller und die Medizin. In: Vitalprinzip Akademie. Festgabe zur 450-Jahrfeier der Universität Jena. Erfurt: Verlag der Akademie Gemeinnütziger Wissenschaften. 2008; S. 17-39.

8 Gutachten aller drei Arbeiten in: NA 41 IIA: 140-3; 176-80.

9 NA 22: 47

10 NA 20: 38-75.

11 Alt (wie Anm. 6): 210.

12 NA 22: 131.

13 NA 23: 39.

14 NA 23: 32

15 NA 23: 15.

16 Dewhurst K, Reeves N. Friedrich Schiller. Medicine, Psychology, Literature. Berkeley, Los Angeles: University of California Press. 1978: 62f.

17 NA 23: 47, 49; NA 24: 3.

18 NA 20: 100.

19 Aristoteles: Poetik. Kap. 6. Zu den medizinischen Hintergründen dieser Theorie: Flashar $\mathrm{H}$. Die medizinischen Grundlagen der Lehre von der Wirkung der Dichtung in der griechischen Poetik. In: Flashar H. Eidola. Ausgewählte kleine Schriften. Amsterdam: Grüner. 1989 S. 109-45. 